\title{
Predicted lean body mass, fat mass, and all cause and cause specific mortality in men: prospective US cohort study
}

\author{
Dong Hoon Lee, ${ }^{1}$ NaNa Keum, ${ }^{1,2}$ Frank B Hu, ${ }^{1,3,4}$ E John Orav, ${ }^{4,5}$ Eric B Rimm, ${ }^{1,3,4}$ \\ Walter C Willett, ${ }^{1,3,4}$ Edward L Giovannucci ${ }^{1,3,4}$
}

${ }^{1}$ Department of Nutrition, Harvard T.H. Chan School of

Public Health, Boston, MA 02115, USA

${ }^{2}$ Department of Food Science and Biotechnology, Dongguk

University, Goyang, South Korea

${ }^{3}$ Department of Epidemiology,

Harvard T.H. Chan School of

Public Health, Boston, MA

02115, USA

${ }^{4}$ Department of Medicine,

Brigham and Women's Hospital and Harvard Medical School,

Boston, MA 02115, USA

${ }^{5}$ Department of Biostatistics,

Harvard T.H. Chan School of

Public Health, Boston, MA

02115, USA

Correspondence to:

E L Giovannucci

egiovann@hsph.harvard.edu

Additional material is published online only. To view please visit

the journal online.

Cite this as: BMJ 2018;362:k2575 http://dx.doi.org/10.1136/bmj.k2575

Accepted: 23 May 2018

\section{ABSTRACT}

OBJECTIVE

To investigate the association of predicted lean body mass, fat mass, and body mass index (BMI) with all cause and cause specific mortality in men.

DESIGN

Prospective cohort study.

SETTING

Health professionals in the United States

PARTICIPANTS

38006 men (aged 40-75 years) from the Health

Professionals Follow-up Study, followed up for death (1987-2012).

MAIN OUTCOME MEASURES

All cause and cause specific mortality.

RESULTS

Using validated anthropometric prediction equations previously developed from the National Health and Nutrition Examination Survey, lean body mass and fat mass were estimated for all participants. During a mean of 21.4 years of follow-up, 12356 deaths were identified. A J shaped association was consistently observed between BMI and all cause mortality. Multivariable adjusted Cox models including predicted fat mass and lean body mass showed a strong positive monotonic association between predicted fat mass and all cause mortality. Compared with those in the lowest fifth of predicted fat mass, men in the highest fifth had a hazard ratio of 1.35 (95\% confidence interval 1.26 to 1.46 ) for mortality from all causes. In contrast, a U shaped association was found between predicted lean body mass and all cause mortality. Compared with those in the lowest fifth of predicted lean body mass, men in the second to fourth fifths had $8-10 \%$ lower risk of mortality from all causes. In the restricted cubic spline models, the risk of all cause mortality was relatively flat until $21 \mathrm{~kg}$ of predicted fat mass and increased rapidly afterwards, with a hazard ratio of 1.22 (1.18 to 1.26) per standard deviation. For predicted lean body mass, a large reduction of the risk was seen within the lower range until $56 \mathrm{~kg}$, with a hazard ratio of $0.87(0.82$ to 0.92$)$ per standard deviation, which increased thereafter ( $P$ for non-linearity $<0.001$ ). For cause specific mortality, men in the highest fifth of predicted fat mass had hazard ratios of 1.67 (1.47 to 1.89) for cardiovascular disease, 1.24 (1.09 to 1.43) for cancer, and 1.26 (0.97 to 1.64) for respiratory disease. On the other hand, a $U$ shaped association was found between predicted lean body mass and mortality from cardiovascular disease and cancer. However, a strong inverse association existed between predicted lean body mass and mortality from respiratory disease ( $P$ for trend $<0.001$ ).

\section{CONCLUSIONS}

The shape of the association between BMI and mortality was determined by the relation between two body components (lean body mass and fat mass) and mortality. This finding suggests that the "obesity paradox" controversy may be largely explained by low lean body mass, rather than low fat mass, in the lower range of BMI.

\section{Introduction}

Obesity is a major public health challenge in the United States and around the world. ${ }^{1}$ In 2013-14, more than two thirds of Americans were classified as overweight (defined as body mass index (BMI) of 25-29.9) or obese (BMI of $\geq 30$ ). ${ }^{2}$ BMI is known as a reasonably good measure of general adiposity, ${ }^{3}$ and many epidemiologic studies have provided evidence showing that obesity, assessed by BMI, is a significant risk factor for increased risk of many chronic diseases as well as mortality. ${ }^{4-6}$ However, the shape of the association between BMI and mortality has been a topic of considerable discussion, as epidemiologic studies have found various types of J shaped, U shaped, and linear relations between BMI and mortality. ${ }^{7}$ For instance, overweight was associated with increased mortality in some studies, ${ }^{8}$ but in others the lowest mortality was observed among overweight people and mortality tended to increase with lower BMI, even after smoking (residual confounding) and pre-existing disease (reverse causation) had been accounted for. ${ }^{910}$ 
This pattern has come to be known as the "obesity paradox." 11 Given the existing and rising number of overweight and obese adults in the US, these divergent findings cause a great deal of confusion among researchers, policy makers, and the general public.

One important but underexplored methodological limitation in the obesity research is that BMI is an imperfect measure of adiposity. ${ }^{12-15}$ Although BMI indicates overweight relative to height, it does not discriminate between fat mass and lean body mass. ${ }^{16-18}$ Body composition is highly variable among individuals with the same BMI. This is particularly important because fat mass and lean body mass may act differently on health outcomes including mortality. Excess fat mass has shown to be detrimental for health, ${ }^{19}$ whereas growing evidence suggests that skeletal muscle, which accounts for most of lean body mass, may be beneficial for health. ${ }^{20}{ }^{21}$ Therefore, understanding the different contributions of lean body mass and fat mass to BMI may provide new insights on the obesity paradox and deliver important clinical and public health messages about healthy body composition beyond BMI. However, direct measurement of lean body mass is particularly difficult in large epidemiologic studies because it requires expensive and sophisticated technologies such as dual energy $x$ ray absorptiometry or imaging technologies. Therefore, little is known about the influence of body composition, particularly lean body mass, on mortality. A limited number of studies have used less accurate surrogate measures (for example, arm circumference, ${ }^{22} 23$ total body potassium,,$^{24}$ skinfold thickness, ${ }^{25}$ and bioelectrical impedance ${ }^{26}$ ) or direct measures to estimate body composition, ${ }^{27-33}$ but these studies had relatively small sample size, short period of follow-up, restricted study population (for example, older people), and/or potential biases (for example, confounding and reverse causation). Moreover, the association of lean body mass and fat mass with cause specific mortality is largely unknown.

Therefore, we used validated anthropometric prediction equations to estimate body composition and examine the association of predicted lean body mass, fat mass, and BMI with all cause and cause specific mortality in a large prospective US cohort study of men. The application of validated equations in a large cohort allowed us to estimate lean body mass and fat mass and examine the independent roles of two different body components in relation to mortality, accounting for potential biases.

\section{Methods}

\section{Study population}

The Health Professionals Follow-up Study started in 1986, when 51529 male health professionals aged 40-75 years were enrolled. Participants were mailed questionnaires at baseline and every two years thereafter to collect updated demographic, lifestyle, and medical information. For the analysis, we included participants who had information on age, race, height, weight, and waist circumference, which we needed to create predicted lean body mass and fat mass ( $n=40226)$. We excluded participants previously diagnosed as having cancer or cardiovascular diseases $(n=1595)$ and those with BMI below 12.5 or above 60 $(n=625)$ at baseline. The final sample size was 38006 men.

\section{Exposure assessments}

Derivation and validation of the predicted lean body mass and fat mass have been described in detail previously. ${ }^{34}$ Briefly, we used a large US representative sample of 7531 men who had measured dual energy $x$ ray absorptiometry from the National Health and Nutrition Examination Survey (NHANES). With lean body mass and fat mass measured by dual energy $x$ ray absorptiometry each as a dependent variable, we did a linear regression using age, race, height, weight, and waist circumference as independent predictors. We then validated the developed equations in an independent validation group of 2292 men and further by using obesity related biomarkers (triglycerides, total cholesterol, high density and low density lipoprotein cholesterol, glucose, insulin, and $\mathrm{C}$ reactive protein). The anthropometric prediction equations (supplementary table A) had high predictive ability for lean body mass $\left(\mathrm{R}^{2}=0.91\right.$, standard error of estimate $2.55 \mathrm{~kg})$ and fat mass $\left(\mathrm{R}^{2}=0.90\right.$, standard error of estimate $2.60 \mathrm{~kg}$ ). In the independent validation group, the actual and predicted lean body mass and fat mass showed robustly high agreement with no evidence of bias. Moreover, the developed equations performed well across different subgroups of the validation group (that is, age, BMI, race, smoking status, and disease status), and predicted fat mass and dual energy $x$ ray absorptiometry measured fat mass showed similar correlations with obesity related biomarkers (Pearson correlations for predicted and measured fat mass, respectively, were $0.33 v 0.34$ for triglycerides, $0.21 v 0.21$ for total cholesterol, $-0.21 v-0.22$ for high density lipoprotein cholesterol, $0.19 v 0.20$ for low density lipoprotein cholesterol, $0.25 v 0.22$ for glucose, $0.51 v 0.54$ for insulin, and $0.31 v 0.32$ for $\mathrm{C}$ reactive protein). ${ }^{34} \mathrm{In}$ an additional validation using the same validation group, dual energy $x$ ray absorptiometry measured values and predicted values showed consistently high agreement with similar errors across the range of lean body mass and fat mass. Scatter plots of the difference between measured and predicted values against measured values showed no strong non-linear pattern (supplementary figures A and B; supplementary tables B and C). For a sensitivity analysis, we also used different prediction equations that include additional polynomial terms of anthropometric measures (supplementary table A). These equations had similar $\mathrm{R}^{2}$ and standard error of estimates but slightly improved fit in the extreme range of lean body mass and fat mass (supplementary figures A and B; supplementary tables B and C). Using the equations, we calculated predicted lean body mass and fat mass for each cohort member on the basis of their age, race, 
height, weight, and waist circumference. Predicted lean body mass and fat mass were available in 1987 , 1996, and 2008.

We collected information on height at enrollment in 1986 and on weight from biennial questionnaires. Distinct from the biennial questionnaire, participants were asked to measure and report their waist circumferences to the nearest quarter inch using provided tape measures and following the same instructions in 1987, 1996, and 2008. Non-responders received follow-up mailings to increase the response rate. In our validation study, the correlation between self reported and technician measured weight and waist circumference were 0.97 and 0.95 , respectively. ${ }^{35}$

\section{Ascertainment of outcomes}

Deaths were identified by reports from the next of kin or postal authorities or by searching the National Death Index. More than $98 \%$ of deaths were ascertained from the follow-up. Cause of death was determined by physicians reviewing medical records and death certificates. ICD-8 (international classification of diseases, 8th revision) codes were used to classify deaths from cardiovascular disease (codes 390-459, 795), cancer (codes 140-239), respiratory disease (codes 460-519), and other causes.

\section{Ascertainment of covariates}

Detailed information on age, race, smoking, and physical activity were collected in 1986 and updated every two years from biennial questionnaires. Family history of cardiovascular disease and cancer were assessed periodically. Dietary information was collected via validated food frequency questionnaires every four years. The Alternate Healthy Eating Index was calculated as an overall measure of diet quality. ${ }^{36}$

\section{Statistical analyses}

We calculated a Spearman correlation between predicted lean body mass and fat mass. We calculated person time of follow-up from the age at which the baseline predicted lean body mass and fat mass were available until the age at death or the end of study (January 2012), whichever came first. We used Cox proportional hazards models to estimate hazard ratios and 95\% confidence intervals. We stratified the analysis by age in months and calendar year of the questionnaire cycle.

We categorized predicted fat mass and lean body mass into fifths on the basis of the distribution of exposures. We used predefined cut points for BMI (<18.5, 18.5-20.4, 20.5-22.4, 22.5-24.9, 25-27.4, 27.5-29.9, 30-34.9, and $\geq 35$ ). For the main analysis, we used predicted fat mass, lean body mass, and BMI measured at baseline to minimize the effect of underlying diseases on mortality. To account for variation in body size, we adjusted for height by including it as a continuous variable in the models with predicted fat mass. As lean body mass is largely determined by height, and thus highly correlated with it, we adjusted for residuals from the regression of predicted lean body mass on height to better capture lean body mass (mostly muscle mass) not related to height. In multivariable models, we adjusted for potential confounders including race, family history of cardiovascular disease, family history of cancer, smoking status, physical activity, total energy intake, alcohol consumption, and Alternate Healthy Eating Index. To examine the independent association of predicted lean body mass and fat mass with mortality, we further ran a multivariable model including both predicted lean body mass and fat mass. Height was adjusted using the same approach described above for lean body mass and fat mass in the mutually adjusted models. We tested for trend by treating the categorical predicted scores and BMI as continuous variables in the model after assigning a median value for each category.

We also used restricted cubic splines with five knots at the 5th, 35th, 50th, 65th, and 95th centiles to flexibly model the association of lean body mass, fat mass, and BMI with mortality. In the spline models, lean body mass and fat mass were mutually adjusted. We tested for potential non-linearity by using a likelihood ratio test comparing the model with only a linear term against the model with linear and cubic spline terms. ${ }^{37-39}$ As the associations of predicted lean body mass, fat mass, and BMI were approximately log linear below and above their medians, we additionally used a linear model to calculate hazard ratios per standard deviation increase in predicted lean body mass, fat mass, and BMI. Given our a priori hypothesis that people with low lean body mass in the lower BMI range cause the $\mathrm{J}$ or $\mathrm{U}$ shaped relation between BMI and mortality, we examined how the shape of BMI-mortality association changes after we excluded participants with low lean body mass (defined as those below the 2.5 th, 5th, and 10th centiles of total participants). For a sensitivity analysis, we additionally examined the shape of BMI-mortality relation after excluding those with low fat mass, using the same criteria as for lean body mass.

To evaluate the latency between predicted lean body mass and fat mass and mortality, we did analyses using different lag times (approximately 0, 4+, 8+, and $12+$ years). For each lagged analysis, the baseline was shifted to $1987,1990,1994$, and 1998, respectively, and predicted lean body mass and fat mass were updated using three repeated measures accordingly. For example, for the no lag time analysis (simple updated), we used the most updated predicted lean body mass and fat mass that were closest to the time of death. For a lag time of 4+ years, we used predicted measures in 1987 for follow-up from 1990 to 2000 and predicted measures in 1996 for follow-up from 2000 to 2012. Similarly, for a lag time of $8+$ years, we used predicted measures in 1987 for deaths in 19942004 and predicted measures in 1996 for deaths in 2004-12. Moreover, we did stratified analyses to explore whether the association of predicted lean body mass and fat mass with mortality varied across smoking status and age. 
We did several sensitivity analyses with no adjustment for physical activity, exclusion of deaths that occurred early in the follow-up period (two years) and right censoring criteria for age ( $>85$ years), and inclusion of baseline illness. We also did analyses using different categories for predicted lean body mass, fat mass, and BMI (that is, fifths and tenths). Lastly, we tested the robustness of our findings by using other prediction equations with polynomial terms. All statistical tests were two sided, and we considered $\mathrm{P}<0.05$ to determine statistical significance. We used SAS 9.4 for all analyses.

\section{Patient involvement}

No patients were involved in setting the research question or the outcome measures, nor were they involved in the design and implementation of the study. There are no plans to involve patients in dissemination.

\section{Results}

\section{Study participants}

We included 38006 men in the analyses. Table 1 shows the baseline characteristics of participants according to BMI categories. The mean age was 54.4 years, and the mean BMI was 25.4. Predicted lean body mass increased with higher BMI. Predicted fat mass slightly deceased in the second category of BMI (18.5-20.4) and then increased with higher BMI. Moreover, men with lower BMI tended to have higher physical activity and Alternate Healthy Eating Index score, peaking in the third category of BMI (20.5-22.4). Although the number of men with underweight (BMI <18.5) was small, they were taller and had lower physical activity and Alternate Healthy Eating Index score than men with normal weight (BMI 18.5-24.9). The Spearman correlation between predicted lean body mass and fat mass was 0.66 in men.

\section{All cause mortality}

During up to 25 years of follow-up (mean 21.4 years), we identified 12356 deaths. Table 2 shows the association of predicted fat mass and lean body mass with all cause mortality in men. A multivariable adjusted model showed a positive association between predicted fat mass and all cause mortality, whereas predicted lean body mass showed a U shaped association with all cause mortality. In a mutually adjusted model including both predicted fat mass and lean body mass, we consistently observed a strong positive association between predicted fat mass and all cause mortality. Compared with those in the lowest fifth of predicted fat mass, men in the highest fifth had a hazard ratio of 1.35 (95\% confidence interval 1.26 to 1.46$)$ for death from any cause. Moreover, predicted lean body mass showed a stronger U shaped association with all cause mortality in the mutually adjusted model. Compared with those in the lowest fifth of predicted lean body mass, men in the second to fourth fifths had 8-10\% lower hazard of death from any cause.

In figure 1, we used restricted cubic splines to flexibly model and visualize the relation of predicted fat mass and lean body mass with all cause mortality in men. The risk of all cause mortality was relatively flat until around $21 \mathrm{~kg}$ of predicted fat mass and then started to increase rapidly afterwards ( $\mathrm{P}$ for non-linearity $<0.001)$. The average BMI for men with $21 \mathrm{~kg}$ of predicted fat mass was 25 . Above $21 \mathrm{~kg}$, the hazard ratio per standard deviation higher predicted fat mass was 1.22 (1.18 to 1.26). Regarding the strong $\mathrm{U}$ shaped relation between predicted lean body mass and all cause mortality, the plot showed a substantial reduction of the risk within the lower range of predicted lean body mass, which reached the lowest risk around $56 \mathrm{~kg}$ and then increased thereafter ( $\mathrm{P}$ for non-linearity $<0.001)$. Below $56 \mathrm{~kg}$, the hazard ratio per standard

\begin{tabular}{|c|c|c|c|c|c|c|c|c|}
\hline \multirow[b]{2}{*}{ Characteristics } & \multicolumn{8}{|c|}{ Body mass index } \\
\hline & $<18.5$ & $18.5-20.4$ & $20.5-22.4$ & $22.5-24.9$ & $25.0-27.4$ & $27.5-29.9$ & $30.0-34.9$ & $\geq 35.0$ \\
\hline Person years & 1839 & 15337 & 92790 & 254122 & 243335 & 95023 & 52320 & 8275 \\
\hline Age, years* & $55.5(10.4)$ & $54.0(10.8)$ & $53.8(10.2)$ & $54.0(9.9)$ & $54.5(9.7)$ & $54.9(9.6)$ & $55.1(9.4)$ & $55.5(10.1)$ \\
\hline Height, $\mathrm{cm}$ & $185.4(12.7)$ & $179.4(7.8)$ & $178.7(6.2)$ & $178.5(6.4)$ & $178.2(6.5)$ & $178.7(6.8)$ & $178.5(7.1)$ & $176.4(10.0)$ \\
\hline Weight, kg & $60.8(8.2)$ & $64.1(5.7)$ & $69.6(5.1)$ & $75.9(5.8)$ & $82.9(6.4)$ & $91.4(7.3)$ & $101.2(9.0)$ & $118.2(13.9)$ \\
\hline Waist circumference, $\mathrm{cm}$ & $86.6(12.4)$ & $82.8(5.3)$ & $86.8(5.1)$ & $91.2(5.6)$ & $96.7(6.1)$ & $102.9(6.8)$ & $110.6(7.9)$ & $123.4(11.4)$ \\
\hline Body mass index & $17.6(0.8)$ & $19.8(0.5)$ & $21.7(0.5)$ & $23.7(0.7)$ & $26.0(0.7)$ & $28.5(0.7)$ & $31.7(1.3)$ & $37.9(3.6)$ \\
\hline Predicted fat mass, $\mathrm{kg}$ & $13.3(5.0)$ & $13.1(2.5)$ & $15.9(2.4)$ & $19.1(2.6)$ & $22.8(2.9)$ & $27.1(3.3)$ & $32.3(4.1)$ & $41.2(6.5)$ \\
\hline Predicted lean body mass, $\mathrm{kg}$ & $40.4(5.8)$ & $47.5(2.2)$ & $50.6(1.9)$ & $53.9(2.1)$ & $57.4(2.3)$ & $61.2(2.6)$ & $65.9(3.4)$ & $75.2(6.0)$ \\
\hline Total energy intake, kcal/day & $2132(610)$ & $2023(570)$ & $2045(599)$ & $2002(595)$ & $1992(609)$ & $2002(625)$ & $2036(639)$ & $2089(657)$ \\
\hline Alcohol consumption, g/day & $14.2(18.7)$ & $9.7(14.2)$ & $10.9(14.3)$ & $11.5(14.7)$ & $11.8(15.4)$ & $11.7(15.5)$ & $10.9(16.1)$ & $8.9(15.1)$ \\
\hline AHEI score & $51.4(13.5)$ & $54.1(12.7)$ & $54.3(12.0)$ & $53.8(11.6)$ & $52.4(11.1)$ & $51.5(10.9)$ & $50.7(11.0)$ & $49.3(10.8)$ \\
\hline Physical activity, MET-h/week & $21.4(35.8)$ & $22.6(27.0)$ & $24.2(28.6)$ & $22.3(27.4)$ & $19.4(23.9)$ & $16.8(22.0)$ & $14.4(20.9)$ & $11.7(14.9)$ \\
\hline White (\%) & 98.4 & 99.2 & 99.5 & 99.3 & 99.2 & 98.8 & 98.7 & 99.4 \\
\hline Family history of CVD (\%) & 35.3 & 32.2 & 33.0 & 33.4 & 33.7 & 33.8 & 35.2 & 35.5 \\
\hline Family history of cancer (\%) & 17.6 & 16.8 & 17.2 & 16.8 & 17.5 & 16.9 & 16.8 & 15.4 \\
\hline \multicolumn{9}{|l|}{ Smoking status (\%): } \\
\hline Never & 47.4 & 56.5 & 56.0 & 50.5 & 45.8 & 44.1 & 42.3 & 41.1 \\
\hline Past & 34.0 & 32.3 & 35.2 & 42.2 & 46.0 & 47.5 & 50.0 & 50.6 \\
\hline Current & 18.6 & 11.2 & 8.9 & 7.3 & 8.2 & 8.4 & 7.8 & 8.3 \\
\hline
\end{tabular}

$\mathrm{AHEl}=$ alternate healthy eating index; $\mathrm{CVD}=$ cardiovascular disease; $\mathrm{MET}=$ metabolic equivalent of task.

*Value is not age adjusted. 


\begin{tabular}{|c|c|c|c|c|c|}
\hline & \multirow[b]{2}{*}{ No of deaths } & \multirow{2}{*}{$\begin{array}{l}\text { Incidence rate per } \\
100000 \text { person years }\end{array}$} & \multicolumn{3}{|l|}{ Hazard ratio $(95 \% \mathrm{Cl})$} \\
\hline & & & Model 1* & Model $2 \dagger$ & Model 3‡ \\
\hline \multicolumn{6}{|l|}{ Fifth of fat mass $\S$} \\
\hline 1 (lowest) & 1937 & 1265 & 1 (reference) & 1 (reference) & 1 (reference) \\
\hline 2 & 2298 & 1504 & 1.09 (1.03 to 1.16$)$ & 1.06 (1.00 to 1.12$)$ & 1.08 (1.01 to 1.15$)$ \\
\hline 3 & 2297 & 1504 & 1.03 (0.97 to 1.09) & $0.98(0.92$ to 1.04$)$ & 1.01 (0.94 to 1.07$)$ \\
\hline 4 & 2726 & 1789 & $1.23(1.16$ to 1.31$)$ & $1.13(1.06$ to 1.20$)$ & $1.16(1.09$ to 1.24$)$ \\
\hline 5 (highest) & 3098 & 2038 & $1.51(1.42$ to 1.60$)$ & $1.33(1.25$ to 1.41$)$ & 1.35 (1.26 to 1.46$)$ \\
\hline$P$ value for trend & & & $<0.001$ & $<0.001$ & $<0.001$ \\
\hline \multicolumn{6}{|c|}{ Fifth of lean body mass $\S$} \\
\hline 1 (lowest) & 2996 & 1969 & 1 (reference) & 1 (reference) & 1 (reference) \\
\hline 2 & 2419 & 1585 & 0.93 (0.88 to 0.98$)$ & 0.93 (0.88 to 0.98$)$ & 0.92 (0.87 to 0.97$)$ \\
\hline 3 & 2324 & 1521 & $0.95(0.90$ to 1.01$)$ & 0.93 (0.88 to 0.98$)$ & 0.90 (0.85 to 0.96$)$ \\
\hline 4 & 2282 & 1494 & 1.03 (0.98 to 1.09$)$ & 1.00 (0.95 to 1.06$)$ & 0.92 (0.87 to 0.98$)$ \\
\hline 5 (highest) & 2335 & 1529 & $1.26(1.20$ to 1.34$)$ & 1.16 (1.10 to 1.23$)$ & 0.97 (0.91 to 1.04$)$ \\
\hline P value for trend & - & - & $<0.001$ & $<0.001$ & 0.49 \\
\hline
\end{tabular}

*Adjusted for age.

tAdjusted for age, race (white or non-white), family history of cardiovascular disease (yes or no), family history of cancer (yes or no), physical activity («3, 3-8.9, 9-17.9, 18-26.9, or >27 MET-h/week), alcohol consumption (0, 0.1-4.9, 5-9.9, 10-14.9, or $\geq 15 \mathrm{~g} /$ day), total energy intake (fifths), smoking status (never, ever, 1-14, 15-24, or $\geq 25$ cigarettes/day), and Alternate Healthy Eating Index (fifths).

¥Additionally, mutually adjusted for predicted fat mass and predicted lean body mass.

$\S$ Derived from validated anthropometric prediction equations; height was adjusted by inclusion as continuous variable for fat mass and by regression out of variation due to height for lean body mass.

deviation higher predicted lean body mass was 0.87 (0.82 to 0.92).

When we used BMI alone, we observed a J shaped association between BMI and all cause mortality in men (table 3 and fig 1). We also examined the influence on BMI after excluding participants with low predicted lean body mass. When we excluded participants below the 2.5 th centile of predicted lean body mass, the J shaped association between BMI and mortality disappeared. After exclusion of more participants with low predicted lean body mass (below 5 th and 10th centiles), the BMI-mortality association became more linear and slightly stronger. However, the J shaped association still existed when we excluded those with low predicted fat mass (supplementary table D).

We further examined how the association of predicted fat mass and lean body mass with all cause mortality changed by different lag times (supplementary table
E). With shorter lag times, predicted fat mass showed a less linear positive association with all cause mortality, whereas predicted lean body mass showed a stronger $\mathrm{U}$ shaped association with all cause mortality. We also examined the associations stratified by smoking status and age (supplementary tables $\mathrm{F}$ and $\mathrm{G}$ ). The association between predicted fat mass and all cause mortality was stronger and more linear among never smokers than among current smokers and stronger among younger adults than among older adults. On the other hand, we observed a stronger $U$ shaped association between predicted lean body mass and all cause mortality among current smokers than among never smokers or past smokers. We observed a similar $\mathrm{U}$ shaped association for predicted lean body mass across all age groups.

Our findings remained robust in several sensitivity analyses (supplementary tables H, I, and J). The

\begin{tabular}{|c|c|c|c|c|c|c|c|}
\hline \multirow[b]{2}{*}{ BMI } & \multirow[b]{2}{*}{ No of deaths } & \multirow{2}{*}{$\begin{array}{l}\text { Incidence rate per } \\
100000 \text { person years }\end{array}$} & \multicolumn{5}{|l|}{ Hazard ratio $(95 \% \mathrm{Cl})$} \\
\hline & & & Model 1* & Model 2* & Model 3t & Model 4‡ & Model 5§ \\
\hline$<18.5$ & 53 & 2883 & 1.74 (1.33 to 2.28$)$ & 1.65 (1.25 to 2.16$)$ & NA & NA & NA \\
\hline $18.5-20.4$ & 269 & 1754 & $1.16(1.03$ to 1.32$)$ & $1.09(0.96$ to 1.24$)$ & 0.95 (0.76 to 1.18$)$ & 0.95 (0.68 to 1.32$)$ & 0.79 (0.38 to 1.66$)$ \\
\hline $20.5-22.4$ & 1358 & 1464 & 1.01 (0.95 to 1.08$)$ & 1.03 (0.97 to 1.09$)$ & 1.01 (0.94 to 1.08$)$ & 0.99 (0.92 to 1.06$)$ & $0.93(0.85$ to 1.03$)$ \\
\hline $22.5-24.9$ & 3740 & 1472 & 1 (reference) & 1 (reference) & 1 (reference) & 1 (reference) & 1 (reference) \\
\hline $25.0-27.4$ & 3986 & 1638 & 1.09 (1.04 to 1.14$)$ & 1.04 (0.99 to 1.09$)$ & 1.04 (1.00 to 1.09$)$ & 1.05 (1.00 to 1.10$)$ & $1.06(1.01$ to 1.11$)$ \\
\hline $27.5-29.9$ & 1753 & 1845 & 1.31 (1.24 to 1.39$)$ & 1.21 (1.14 to 1.28$)$ & $1.22(1.15$ to 1.29$)$ & $1.23(1.16$ to 1.30$)$ & $1.24(1.17$ to 1.31$)$ \\
\hline $30.0-34.9$ & 1001 & 1913 & $1.48(1.38$ to 1.59$)$ & 1.31 (1.22 to 1.41$)$ & 1.31 (1.22 to 1.41$)$ & 1.33 (1.24 to 1.43$)$ & 1.34 (1.25 to 1.44$)$ \\
\hline$\geq 35.0$ & 196 & 2368 & 2.28 (1.98 to 2.64$)$ & 2.01 (1.74 to 2.33 ) & 2.02 (1.75 to 2.34$)$ & 2.04 (1.76 to 2.36$)$ & 2.06 (1.78 to 2.38$)$ \\
\hline$P$ value for trend & - & - & $<0.001$ & $<0.001$ & $<0.001$ & $<0.001$ & $<0.001$ \\
\hline
\end{tabular}

$\mathrm{NA}=$ not available (no cases available after exclusion).

*Model 1 adjusted for age. Model 2 adjusted for age, race (white or non-white), family history of cardiovascular disease (yes or no), family history of cancer (yes or no), physical activity ( $<3,3-8.9$, 9-17.9, 18-26.9, or $>27 \mathrm{MET}$-h/week), alcohol consumption (0, 0.1-4.9, 5-9.9, 10-14.9, or $\geq 15 \mathrm{~g} /$ day), total energy intake (fifths), smoking status (never, ever, 1-14, 15-24, or $\geq 25$ cigarettes/ day), and Alternate Healthy Eating Index (fifths). No of deaths/person years for each category of BMI: 53/1839, 269/15337, 1358/92 790, 3740/254 122, 3986/243 335, 1753/95 023, $1001 / 52320$, and $196 / 8275$.

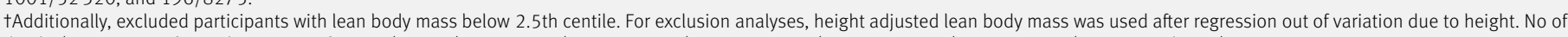
deaths/person years for each category of BMI: 0/26, 80/7196, 1147/85 194, 3695/252 987, 3980/243212, 1751/94 960, 1000/52 311, and 196/8275.

fAdditionally, excluded participants with lean body mass below 5 th centile. For exclusion analyses, height adjusted lean body mass was used after regression out of variation due to height. No of deaths/person years for each category of BMI: 0/0, 36/3402, 884/72 686, 3603/250 459, 3976/243080, 1751/94960, 1000/52 311, and 196/8275.

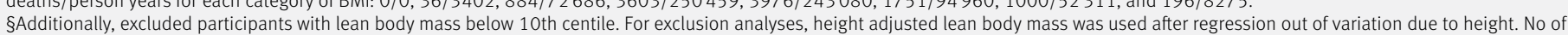
deaths/person years for each category of BMI: 0/0, 7/781, 509/48989, 3295/239196, 3963/242 606, 1751/94928, 1000/52311, and 196/8275. 
results did not change with no adjustment for physical activity, exclusion of deaths early in the follow-up period and right censoring criteria for age, inclusion of baseline illness, or use of fifths and tenths for
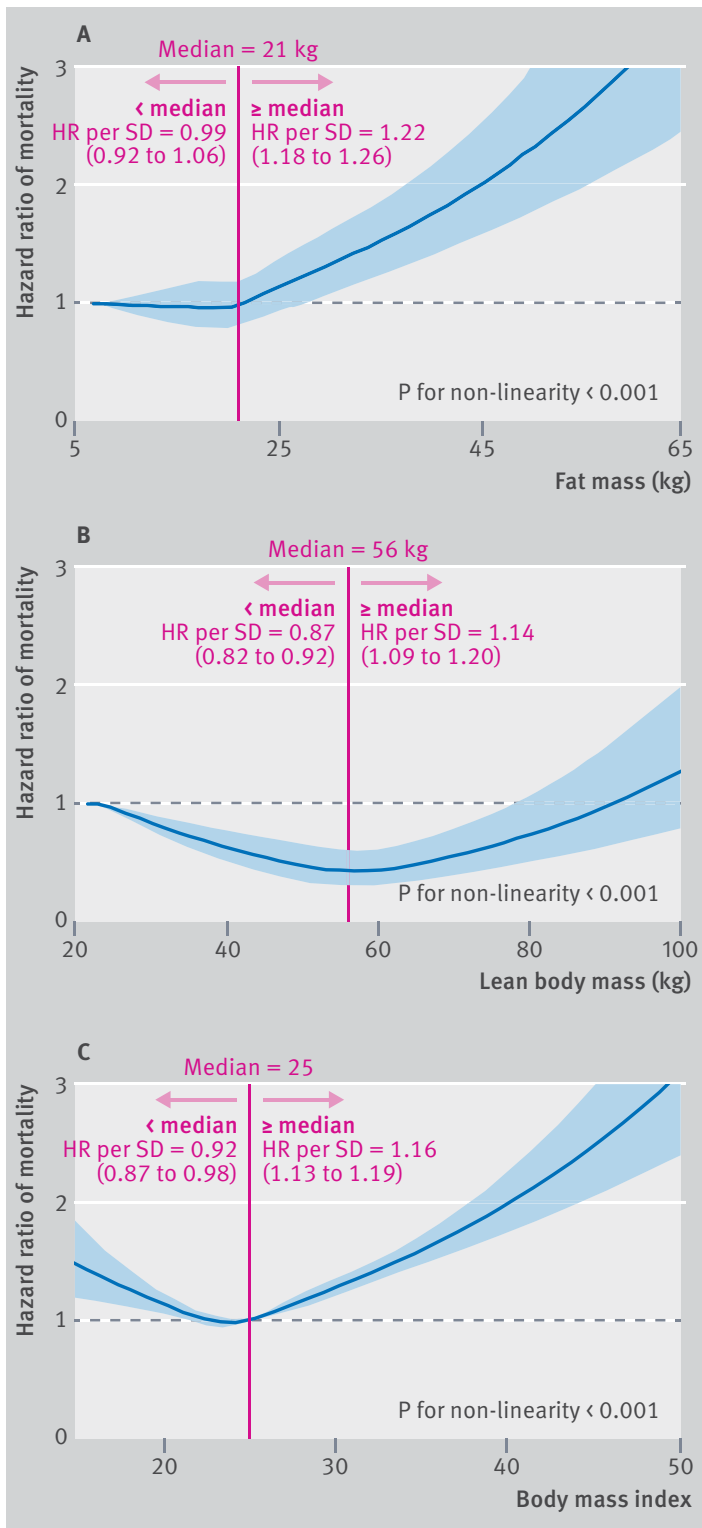

Fig 1 | Association of predicted body composition and body mass index $(\mathrm{BMI})^{\star}$ with all cause mortality in men. A: Fat mass and all cause mortality. B: Lean body mass and all cause mortality. C: BMI and all cause mortality. Hazard ratios are indicated by solid lines and $95 \% \mathrm{Cls}$ by shaded areas. Reference point is lowest value for each of fat mass and lean body mass and 25 for BMI, with knots placed at 5 th, 35th, 50th, 65 th, and 95 th centiles of each fat mass, lean body mass, and BMI distribution. All models were adjusted for cofounders in table 2 . For graphs A and B, predicted fat mass and predicted lean body mass were mutually adjusted. *Standard deviations: $5.7 \mathrm{~kg}$ for fat mass, $6.6 \mathrm{~kg}$ for lean body mass, and 3.1 for BMI; centiles $(0,2.5,5,10,25,50,75$, 90, and 100): 7, 13, 14, 15, 18, 21, 25, 29, and $66 \mathrm{~kg}$ for fat mass; $24,48,49,51,53,56,59,63$, and $103 \mathrm{~kg}$ for lean body mass; and 14.2, 20.5, 21.2, 22.0, 23.4, 25.1, $27.0,29.3$, and 62.0 for BMI exposures. Moreover, use of other prediction equations with polynomial terms showed consistent results (data not shown but available on request).

\section{Cause specific mortality}

We further examined the association of predicted fat mass and lean body mass with cause specific mortality (table 4). Mutually adjusted models showed a linear positive association between predicted fat mass and mortality from cardiovascular disease and cancer. Compared with those in the lowest fifth of predicted fat mass, men in the highest fifth had hazard ratios of 1.67 (1.47 to 1.89) for death from cardiovascular disease, 1.24 (1.09 to 1.43) for death from cancer, and 1.26 (0.97 to 1.64) for death from respiratory disease. In contrast, predicted lean body mass showed a U shaped association with mortality from cardiovascular disease and cancer in the mutually adjusted models. However, predicted lean body mass showed a strong inverse association with mortality from respiratory disease $(\mathrm{P}$ for trend $<0.001)$. Compared with those in the lowest fifth of predicted lean body mass, men in the highest fifth had a hazard ratio of 0.50 (0.39 to 0.65$)$ for death from respiratory disease. When we examined the association between BMI and cause specific mortality, we observed a $U$ shaped association for death from cardiovascular disease but a positive association for death from cancer and an inverse association for death from respiratory disease.

\section{Discussion}

In a large prospective cohort study of men, we used validated anthropometric prediction equations to examine the association of lean body mass and fat mass with all cause and cause specific mortality. We found a strong positive association between predicted fat mass and mortality from all causes, cardiovascular disease, and cancer. In contrast, predicted lean body mass showed a U shaped association with mortality from all causes, cardiovascular disease, and cancer, and an inverse association with mortality from respiratory disease.

\section{Comparison with other studies}

Many epidemiologic studies have examined the relation between BMI and mortality, but confusion exists around the unexpected J shaped or U shaped association observed. ${ }^{8} 9$ A systematic review and meta-analysis of 97 prospective studies in 2013 reported that, relative to normal weight (BMI 18.524.9), obesity (BMI $\geq 30$ ) was associated with higher all cause mortality, but overweight (BMI 25-29.9) was associated with lower all cause mortality. ${ }^{9}$ In contrast, a meta-analysis of data from individual participants in 239 prospective studies by the Global BMI Mortality Collaboration in 2016 found evidence that increased risk of all cause mortality among overweight people was largely due to confounding by smoking and reverse causation from underlying disease and frailty at older ages. ${ }^{8}$ Controversies are ongoing around the "obesity paradox, ${ }^{, 40-42}$ with many studies still reporting excess 


\begin{tabular}{|c|c|c|c|c|}
\hline & \multicolumn{4}{|l|}{ Hazard ratio $(95 \% \mathrm{Cl})$} \\
\hline & Cardiovascular disease death & Cancer death & Respiratory death & Other death \\
\hline No of deaths & 4297 & 3726 & 960 & 3373 \\
\hline $\begin{array}{l}\text { Incidence rate per } 100000 \\
\text { person years }\end{array}$ & 558 & 483 & 124 & 437 \\
\hline \multicolumn{5}{|l|}{ Fifth of fat mass ${ }^{\star}$} \\
\hline 1 (lowest) & 1 (reference) & 1 (reference) & 1 (reference) & 1 (reference) \\
\hline 2 & 1.11 (0.99 to 1.24$)$ & 1.15 (1.03 to 1.29$)$ & $0.92(0.74$ to 1.14$)$ & 1.01 (0.90 to 1.14$)$ \\
\hline 3 & 1.10 (0.98 to 1.23$)$ & 1.06 (0.94 to 1.19$)$ & $1.06(0.85$ to 1.31$)$ & 0.84 (0.74 to 0.94$)$ \\
\hline 4 & $1.30(1.16$ to 1.46$)$ & 1.15 (1.02 to 1.30$)$ & 1.10 (0.88 to 1.38$)$ & $1.02(0.90$ to 1.15$)$ \\
\hline 5 (highest) & 1.67 (1.47 to 1.89$)$ & $1.24(1.09$ to 1.43$)$ & $1.26(0.97$ to 1.64$)$ & 1.13 (0.98 to 1.30$)$ \\
\hline P value for trend & $<0.001$ & 0.005 & 0.03 & 0.05 \\
\hline \multicolumn{5}{|l|}{ Lean body mass ${ }^{\star}$} \\
\hline 1 (lowest) & 1 (reference) & 1 (reference) & 1 (reference) & 1 (reference) \\
\hline 2 & $0.96(0.88$ to 1.06$)$ & 0.97 (0.88 to 1.08$)$ & $0.61(0.51$ to 0.74$)$ & $0.94(0.84$ to 1.04$)$ \\
\hline 3 & 0.95 (0.86 to 1.05$)$ & $0.94(0.84$ to 1.04$)$ & $0.58(0.47$ to 0.71$)$ & 0.95 (0.86 to 1.07$)$ \\
\hline 4 & $0.96(0.87$ to 1.07$)$ & $0.95(0.85$ to 1.06$)$ & $0.57(0.46$ to 0.71$)$ & $1.00(0.89$ to 1.12$)$ \\
\hline 5 (highest) & $1.11(0.99$ to 1.24$)$ & $1.02(0.90$ to 1.16$)$ & $0.50(0.39$ to 0.65$)$ & 0.98 (0.86 to 1.12$)$ \\
\hline$P$ value for trend & 0.10 & 0.89 & $<0.001$ & 0.99 \\
\hline \multicolumn{5}{|l|}{ Body mass index } \\
\hline$<18.5$ & $1.45(0.87$ to 2.41$)$ & $0.66(0.32$ to 1.40$)$ & $5.33(3.10$ to 9.17$)$ & $1.86(1.15$ to 3.01$)$ \\
\hline $18.5-20.4$ & $1.12(0.90$ to 1.38$)$ & $0.99(0.78$ to 1.25$)$ & $1.93(1.36$ to 2.73$)$ & $0.92(0.72$ to 1.19$)$ \\
\hline $20.5-22.4$ & $0.95(0.85$ to 1.06$)$ & 0.97 (0.87 to 1.09$)$ & $1.30(1.06$ to 1.60$)$ & 1.09 (0.97 to 1.22$)$ \\
\hline $22.5-24.9$ & 1 (reference) & 1 (reference) & 1 (reference) & 1 (reference) \\
\hline $25.0-27.4$ & $1.16(1.08$ to 1.26$)$ & $1.01(0.93$ to 1.09$)$ & 0.91 (0.78 to 1.08$)$ & $0.96(0.88$ to 1.05$)$ \\
\hline $27.5-29.9$ & $1.40(1.27$ to 1.54$)$ & $1.13(1.02$ to 1.25$)$ & 1.09 (0.89 to 1.35$)$ & $1.10(0.98$ to 1.23$)$ \\
\hline $30.0-34.9$ & 1.75 (1.56 to 1.96$)$ & $1.12(0.98$ to 1.28$)$ & 0.81 (0.60 to 1.09$)$ & $1.18(1.02$ to 1.35$)$ \\
\hline$\geq 35.0$ & $2.66(2.11$ to 3.36$)$ & 1.55 (1.17 to 2.04$)$ & $0.90(0.43$ to 1.92$)$ & 2.13 (1.63 to 2.77$)$ \\
\hline$P$ value for trend & $<0.001$ & $<0.001$ & $<0.001$ & 0.001 \\
\hline \multicolumn{5}{|c|}{$\begin{array}{l}\text { All models were adjusted for age, race (white or non-white), family history of cardiovascular disease (yes or no), family history of cancer (yes or no), physical } \\
\text { activity ( } 3,3,3-8.9,9-17.9,18-26.9, \text { or } 27 \mathrm{MET}-\mathrm{h} / \text { week), acohol consumption (0, } 0.1-4.9,5-9.9,10-14.9, \text { or } \geq 15 \mathrm{~g} / \text { day), total energy intake (fifths), } \\
\text { smoking status (never, ever, } 1-14,15-24, \text { or } \geq 25 \text { cigarettes/day), and Alternate Healthy Eating Index (fifths). Fat mass and lean body mass were mutually } \\
\text { adjusted in model. } \\
\text { ×Derived from validated anthropometric prediction equations. Height was adjusted by inclusion as continuous variable for fat mass and by regression out of } \\
\text { variation due to height for lean body mass. }\end{array}$} \\
\hline
\end{tabular}

mortality at the lower BMI range. More importantly, these studies acknowledged the major limitation of BMI as a measure of adiposity but did not directly investigate the two distinct compartments of body composition (lean body mass and fat mass) in relation to mortality. ${ }^{89}$

Our findings on BMI were in line with the previous findings, whereby we consistently observed a J shaped association with mortality even after accounting for age, smoking, and baseline diseases. Our study added a new insight that the observed J shaped association between BMI and mortality can be explained when the two different shapes in mortality risk for fat mass and lean body mass are taken together. The increased risk of mortality in the lower BMI range $(<25)$ could be attributed to a combination of the high risk among men with low predicted lean body mass, which overrides the weak association between predicted fat mass and mortality in this lower range of BMI. The increase of mortality risk at the BMI range of 25-29.9 is likely due to the high risk associated with predicted fat mass in combination with only a moderate risk associated with predicted lean body mass. Lastly, the rapid increase in mortality risk in the higher BMI range $(\geq 30)$ could be due to a very high risk associated with both predicted fat mass and lean body mass. Of note, at the high end of BMI ( $\geq 30$ ), the vast majority of people have high predicted fat mass and lean body mass. Those with high predicted lean body mass almost invariably have high fat mass; for example, the average predicted fat mass for those in the highest tenth of predicted lean body mass was $31 \mathrm{~kg}$ (supplementary table J).

These observed patterns for fat mass and lean body mass were further supported by our additional analyses of BMI and mortality after exclusion of participants at the lower end of predicted lean body mass, which resulted in a strong linear positive relation between BMI and mortality. This shows that separating lean and healthy (low BMI and normal lean body mass) from lean and unhealthy (low BMI and low lean body mass) people could be a key to explain the obesity paradox phenomenon. One hypothesis states that accumulating excess fat may be causally beneficial for survivors of cancers or other diseases. ${ }^{43}$ However, our results suggest the possibility that people with more fat stores may seem to be at lower risk than those with lower BMI, but that this may not be causal but rather a result of the comparison group having low lean body mass.

To date, only a limited number of studies have examined mortality in relation to body composition measured directly using dual energy $x$ ray absorptiometry or computed tomography. ${ }^{27-33}$ Most studies have been done in older populations with an approximate mean age of 75 years. ${ }^{27-29} 3133$ The findings showed inconsistent and various shapes of 
the association. An Italian study of 934 older people showed no association of calf muscle and fat mass area with six year mortality, ${ }^{28}$ whereas another study of 2292 older people from the US found that low leg muscle area was associated with increased risk of six year mortality in men only. ${ }^{29}$ In contrast, other studies found that lower fat percentage or leg fat mass was associated with increased mortality, ${ }^{273133}$ but only one of them, in 477 community dwelling older people from the Netherlands, reported that lower appendicular skeletal muscle mass was associated with increased risk of 12 year mortality. ${ }^{33}$ Although these studies used a direct measure of body composition, the study samples were restricted to an older population, which limits the generalizability of their findings. Moreover, they also had other limitations such as small sample size, short follow-up, exposure measured at one time point, lack of information on important confounders (especially smoking), and no examination of cause specific mortality. Nevertheless, our finding was consistent with a recent large scale Canadian study that measured dual energy $x$ ray absorptiometry in participants referred for bone mineral density testing. ${ }^{30}$ That study found that high percentage fat and low BMI were independently associated with increased risk of mortality when percentage fat and BMI were simultaneously adjusted in the models. However, the observed associations might have been confounded by smoking or physical activity owing to a lack of information on those variables, and the study did not directly use lean body mass in the analysis.

The BMI-mortality association is prone to reverse causation by pre-existing diseases that can cause weight loss and also increase risk of mortality, and this is more likely to be a concern with shorter lag times. We found that, with shorter lag time periods, the positive association between predicted fat mass and mortality was attenuated, whereas the $U$ shaped association between predicted lean body mass and mortality tended to be strengthened. Therefore, the stronger $\mathrm{U}$ shaped relation between BMI and mortality with shorter lag time periods can be mostly attributed to the pronounced $U$ shaped association for predicted lean body mass, which may be an indicator of health status capturing any pre-existing undiagnosed medical condition, including frailty at older ages.

The influence of smoking is particularly important in investigating the obesity-mortality association. Not only is smoking a strong risk factor for death, but it also affects body weight and body composition. ${ }^{44-47}$ Similar to the BMI-mortality association, predicted fat mass showed a weaker and less linear association with mortality among current smokers than in past smokers or never smokers. Interestingly, we found a stronger U shaped association between predicted lean body mass and mortality among current smokers than in past or never smokers. Although we cannot completely rule out residual confounding by smoking, our findings showed some evidence that the frequently observed U shaped association between BMI and mortality among smokers may be affected by the strong $U$ shaped association between lean body mass and mortality.

\section{Strengths and limitations of study}

Our study has several strengths. Firstly, the innovative approach of validated anthropometric prediction equations allowed us to estimate lean body mass and fat mass practically in large epidemiologic settings. This is the first analysis to examine the association of predicted body composition with all cause and cause specific mortality in a large prospective cohort study. Secondly, the Health Professionals Follow-up Study is a well established prospective cohort study that has a large number of deaths over a long term follow-up period. Thirdly, detailed and updated information on lifestyle and health related factors allowed adequate control for confounding. Fourthly, repeated measures on exposures (that is, predicted scores) allowed prospective analyses of different lag time periods to examine reverse causality in the obesity-mortality association.

The study also has several limitations. Firstly, predicted lean body mass and fat mass are not perfect measures of actual lean body mass and fat mass. Nevertheless, the validation results from NHANES showed high predictive ability of the anthropometric equations with no systematic bias. In fact, the very high $\mathrm{R}^{2}$ between fat mass and lean body mass $(>0.90)$ for direct dual energy $x$ ray absorptiometry measurements and predicted measures in an independent dataset indicate that a direct dual energy $x$ ray absorptiometry measure would give very similar insights to ours; this is further supported by the equal predictive ability of the predicted measures and dual energy $x$ ray absorptiometry measures for various obesity related biomarkers. Moreover, given the prospective study design, any mismeasurement in the exposures would likely be random with respect to endpoints, resulting in conservative associations. Secondly, we cannot entirely rule out the possibility of unmeasured or unknown confounding factors that may account for the associations observed in this study. However, the homogeneity of the study population and comprehensive data on the risk factors minimized potential confounding. Thirdly, the generalizability of the findings may be limited given that the study participants were restricted to health professionals and predominantly white men. However, we believe that our main findings will be broadly applicable.

\section{Clinical and public health implications}

This study provides strong evidence that excess fat mass increases the risk of mortality. Increased fat mass was not protective for mortality, which is counter to the premise of the "obesity paradox." On the other hand, low lean body mass was associated with an increased risk of mortality in the lower range of BMI. Our study suggests that understanding different contributions of lean body mass and fat mass to BMI may explain the shape of the association between BMI and mortality as well as the controversial matter of the obesity paradox. 
Our findings support recommendations to maintain a normal body weight defined by BMI of 18.5 to 24.9 for prevention of chronic diseases and further suggest that the lowest mortality risk can be observed at the lower normal range of BMI if the influence of lean body mass on mortality is accounted for. The recommendations should highlight not only the importance of normal body weight but also healthy body composition (for example, healthy lean) to reduce confusion around the optimal weight (the obesity paradox) for overall health. Interventions and strategies to promote healthy body composition through lifestyle modification (such as physical activity and diet) may be an important next step to improve the health of the population.

\section{Conclusions}

We found a strong positive association between predicted fat mass and mortality and a $U$ shaped association between predicted lean body mass and mortality in men. Low lean body mass, rather than low fat mass, may be driving the increased risk of mortality in the lower BMI range. Understanding the independent roles of lean body mass and fat mass has important implications for clarifying the obesity paradox phenomenon in the association between BMI and mortality.

Acknowledgements: We thank the participants and staff of the Health Professionals Follow-up Study for their valuable contributions, as well as the following state cancer registries for their help: AL, AZ, AR, CA, CO, CT, DE, FL, GA, ID, IL, IN, IA, KY, LA, ME, MD, MA, MI, NE, NH, NJ, NY, NC, ND, OH, OK, OR, PA, RI, SC, TN, TX, VA, WA, WY. The authors assume full responsibility for analyses and interpretation of these data.

Contributors: DHL and ELG had full access to all of the data in the study and take responsibility for the integrity of the data and the accuracy of the data analysis. DHL and ELG conceived and designed the study. NK, FBH, EBR, WCW, and ELG obtained funding. FBH, EJO, EBR, WCW, and ELG acquired the data. DHL did the statistical analysis. DHL and ELG drafted the manuscript. All the authors critically revised the manuscript for important intellectual content. DHL and ELG were responsible for administrative, technical, or material support. ELG was responsible for study supervision. The corresponding author attests that all listed authors meet authorship criteria and that no others meeting the criteria have been omitted. $\mathrm{DHL}$ is the guarantor.

Funding: This work was supported by the National Institutes of Health (UM1 CA167552, R01 HL35464, and R03 CA223619) and the Basic Research Lab Program through the National Research Foundation of Korea (NRF) funded by the MSIT (NRF-2018R1A4A1022589). The funders had no role in the design and conduct of the study; collection, management, analysis, and interpretation of the data; preparation, review, or approval of the manuscript; or the decision to submit the manuscript for publication

Competing interests: All authors have completed the ICMJE uniform disclosure form at http://www.icmje.org/coi_disclosure.pdf (available on request from the corresponding author) and declare: no support from any organization for the submitted work other than that described above; no financial relationships with any organizations that might have an interest in the submitted work in the previous three years; no other relationships or activities that could appear to have influenced the submitted work.

Ethical approval: This investigation was approved by the Institutional Review Board of the Harvard T.H. Chan School of Public Health and Brigham and Women's Hospital.

Data sharing: No additional data available.

Transparency declaration: The lead author (the manuscript's guarantor) affirms that the manuscript is an honest, accurate, and transparent account of the study being reported; that no important aspects of the study have been omitted; and that any discrepancies from the study as planned (and, if relevant, registered) have been explained

This is an Open Access article distributed in accordance with the Creative Commons Attribution Non Commercial (CC BY-NC 4.0) license, which permits others to distribute, remix, adapt, build upon this work non-commercially, and license their derivative works on different terms, provided the original work is properly cited and the use is noncommercial. See: http://creativecommons.org/licenses/by-nc/4.0/

1 Obesity: preventing and managing the global epidemic. Report of a WHO consultation. World Health Organ Tech Rep Ser 2000;894:i-xii, $1-253$.

2 Centers for Disease Control and Prevention. National Health and Nutrition Examination Survey. 2018. http://www.cdc.gov/nchs/ about/major/nhanes/datalink.htm.

3 Hu F. Obesity epidemiology. Oxford University Press, 2008. doi:10.1093/acprof:oso/9780195312911.001.0001.

4 Adams KF, Schatzkin A, Harris TB, et al. Overweight, obesity, and mortality in a large prospective cohort of persons 50 to 71 years old. N Engl J Med 2006;355:763-78. doi:10.1056/ NEJMoa055643

5 Berrington de Gonzalez A, Hartge P, Cerhan JR, et al. Body-mass index and mortality among 1.46 million white adults. $N$ Engl Med 2010;363:2211-9. doi:10.1056/NEIMoa1000367

6 Chen Z, Yang G, Offer A, et al. Body mass index and mortality in China: a 15-year prospective study of 220000 men. Int J Epidemiol 2012:41:472-81. doi:10.1093/ije/dyr208

7 Manson JE, Bassuk SS, Hu FB, Stampfer MJ, Colditz GA, Willett WC Estimating the number of deaths due to obesity: can the divergent findings be reconciled? J Womens Health (Larchmt) 2007;16:168-76. doi:10.1089/jwh.2006.0080

8 Di Angelantonio E, Bhupathiraju ShN, Wormser D, et al, Global BMI Mortality Collaboration. Body-mass index and all-cause mortality: individual-participant-data meta-analysis of 239 prospective studies in four continents. Lancet 2016;388:776-86. doi:10.1016/S01406736(16)30175-1

9 Flegal KM, Kit BK, Orpana H, Graubard BI. Association of allcause mortality with overweight and obesity using standard body mass index categories: a systematic review and meta-analysis. JAMA 2013;309:71-82. doi:10.1001/jama.2012.113905

10 Veronese $\mathrm{N}$, Cereda E, Solmi $\mathrm{M}$, et al. Inverse relationship between body mass index and mortality in older nursing home residents: a meta-analysis of 19,538 elderly subjects. Obes Rev 2015;16:1001 15. doi:10.1111/obr.12309

11 Greenberg JA. The obesity paradox in the US population. Am J Clin Nutr 2013;97:1195-200. doi:10.3945/ajcn.112.045815

12 Visscher TL, Seidell JC, Molarius A, van der Kuip D, Hofman A, Witteman JC. A comparison of body mass index, waist-hip ratio and waist circumference as predictors of all-cause mortality among the elderly: the Rotterdam study. Int I Obes Relat Metab Disord 2001;25:1730-5. doi:10.1038/sj.ijo.0801787

13 Romero-Corral A, Lopez-Jimenez F, Sierra-Johnson J, Somers VK. Differentiating between body fat and lean mass-how should we measure obesity? Nat Clin Pract Endocrinol Metab 2008;4:322-3. doi:10.1038/ncpendmet0809

14 Okorodudu DO, Jumean MF, Montori VM, et al. Diagnostic performance of body mass index to identify obesity as defined by body adiposity: a systematic review and meta-analysis. Int J Obes (Lond) 2010;34:791-9. doi:10.1038/ijo.2010.5

15 Pischon T. Commentary: Use of the body mass index to assess the risk of health outcomes: time to say goodbye? Int I Epidemiol 2010;39:528-9. doi:10.1093/ije/dyp388

16 Gallagher D, Visser M, Sepúlveda D, Pierson RN, Harris T, Heymsfield SB. How useful is body mass index for comparison of body fatness across age, sex, and ethnic groups? Am J Epidemiol 1996;143:228-39. doi:10.1093/oxfordjournals.aje. a008733

17 Gallagher D, Ruts E, Visser M, et al. Weight stability masks sarcopenia in elderly men and women. Am J Physiol Endocrinol Metab 2000;279:E366-75. doi:10.1152/ ajpendo.2000.279.2.E366

18 Harris TB. Invited commentary: body composition in studies of aging: new opportunities to better understand health risks associated with weight. Am J Epidemiol 2002;156:122-4, discussion 125-6. doi:10.1093/aje/kwf024

19 Haslam DW, James WP. Obesity. Lancet 2005;366:1197-209. doi:10.1016/S0140-6736(05)67483-1

20 Wannamethee SG, Atkins JL. Muscle loss and obesity: the health implications of sarcopenia and sarcopenic obesity. Proc Nutr Soc 2015;74:405-12. doi:10.1017/S002966511500169X

21 Rolland Y, Czerwinski S, Abellan Van Kan G, et al. Sarcopenia: its assessment, etiology, pathogenesis, consequences and future perspectives. J Nutr Health Aging 2008;12:433-50. doi:10.1007/ BF02982704 
22 Wannamethee SG, Shaper AG, Lennon L, Whincup PH. Decreased muscle mass and increased central adiposity are independently related to mortality in older men. Am J Clin Nutr 2007;86:1339-46. doi:10.1093/ajcn/86.5.1339

23 Allison DB, Zhu SK, Plankey M, Faith MS, Heo M. Differential associations of body mass index and adiposity with all-cause mortality among men in the first and second National Health and Nutrition Examination Surveys (NHANES I and NHANES II) follow-up studies. Int J Obes Relat Metab Disord 2002;26:410-6. doi:10.1038/ sj.ijo.0801925

24 Heitmann BL, Erikson H, Ellsinger BM, Mikkelsen KL, Larsson B. Mortality associated with body fat, fat-free mass and body mass index among 60-year-old swedish men-a 22-year followup. The study of men born in 1913. Int J Obes Relat Metab Disord 2000;24:33-7. doi:10.1038/sj.ijo.0801082

25 Lee CD, Blair SN, Jackson AS. Cardiorespiratory fitness, body composition, and all-cause and cardiovascular disease mortality in men. Am J Clin Nutr 1999;69:373-80. doi:10.1093/ ajcn/69.3.373

26 Bigaard J, Frederiksen K, Tjønneland A, et al. Body fat and fatfree mass and all-cause mortality. Obes Res 2004;12:1042-9. doi:10.1038/oby.2004.131

27 Auyeung TW, Lee JS, Leung J, Kwok T, Leung PC, Woo J. Survival in older men may benefit from being slightly overweight and centrally obese--a 5-year follow-up study in 4,000 older adults using DXA. Gerontol A Biol Sci Med Sci 2010;65:99-104. doi:10.1093/gerona/ glp099

28 Cesari M, Pahor M, Lauretani F, et al. Skeletal muscle and mortality results from the InCHIANTI Study. I Gerontol A Biol Sci Med Sci 2009;64:377-84. doi:10.1093/gerona/gln031

29 Newman AB, Kupelian V, Visser M, et al. Strength, but not muscle mass, is associated with mortality in the health, aging and body composition study cohort. J Gerontol A Biol Sci Med Sci 2006;61:72 7. doi:10.1093/gerona/61.1.72

30 Padwal R, Leslie WD, Lix LM, Majumdar SR. Relationship Among Body Fat Percentage, Body Mass Index, and All-Cause Mortality: A Cohort Study. Ann Intern Med 2016;164:532-41. doi:10.7326/M151181

31 Rolland Y, Gallini A, Cristini C, et al. Body-composition predictors of mortality in women aged $\geq 75 \mathrm{y}$ : data from a large population-based cohort study with a 17-y follow-up. Am J Clin Nutr 2014;100:135260. doi:10.3945/ajen.114.086728

32 Toss F, Wiklund P, Nordström P, Nordström A. Body composition and mortality risk in later life. Age Ageing 2012;41:677-81 doi:10.1093/ageing/afs087

33 Wijnhoven HA, Snijder MB, van Bokhorst-de van der Schueren MA, Deeg DJ, Visser M. Region-specific fat mass and muscle mass and mortality in community-dwelling older men and women. Gerontology 2012;58:32-40. doi:10.1159/000324027
34 Lee DH, Keum N, Hu FB, et al. Development and validation of anthropometric prediction equations for lean body mass, fat mass and percent fat in adults using the National Health and Nutrition Examination Survey (NHANES) 1999-2006. Br J Nutr 2017;118:85866. doi:10.1017/S0007114517002665

35 Rimm EB, Stampfer MJ, Colditz GA, Chute CG, Litin LB, Willett WC Validity of self-reported waist and hip circumferences in men and women. Epidemiology 1990;1:466-73. doi:10.1097/00001648199011000-00009

36 McCullough ML, Willett WC. Evaluating adherence to recommended diets in adults: the Alternate Healthy Eating Index. Public Health Nutr 2006;9(1A):152-7. doi:10.1079/PHN2005938

37 Durrleman S, Simon R. Flexible regression models with cubic splines. Stat Med 1989;8:551-61. doi:10.1002/sim.4780080504

38 Govindarajulu U, Spiegelman D, Thurston S, Eisen E. Comparing smoothing techniques for modeling exposure-response curves in Cox models. Stat Med 2007;26:52. doi:10.1002/sim.2848.

39 Smith PL. Splines as a useful and convenient statistical tool. Am Stat 1979;33:57-62

40 Flegal KM, Ioannidis JPA. A meta-analysis but not a systematic review: an evaluation of the Global BMI Mortality Collaboration. J Clin Epidemiol 2017;88:21-9. doi:10.1016/j.jclinepi.2017.04.007

41 Bhupathiraju SN, Di Angelantonio E, Danesh J, Hu FB. Commentary on "A meta-analysis but not a systematic review: an evaluation of the Global BMI Mortality Collaboration”. J Clin Epidemiol 2017;88:30-2. doi:10.1016/j.jclinepi.2017.04.006

42 Flegal KM, loannidis JPA. A meta-analysis of individual participant data constructed to align with prior expert views: comments on Bhupathiraju et al. J Clin Epidemiol 2017;88:33-6. doi:10.1016/j. jclinepi.2017.04.005

43 Tobias DK. Addressing Reverse Causation Bias in the Obesity Paradox Is Not “One Size Fits All”. Diabetes Care 2017;40:1000-1. doi:10.2337/dci17-0010

44 Chiolero A, Faeh D, Paccaud F, Cornuz J. Consequences of smoking for body weight, body fat distribution, and insulin resistance. Am J Clin Nutr 2008;87:801-9. doi:10.1093/ajcn/87.4.801

45 Molarius A, Seidell JC, Kuulasmaa K, Dobson AJ, Sans S. Smoking and relative body weight: an international perspective from the WHO MONICA Project. J Epidemiol Community Health 1997;51:252-60. doi:10.1136/jech.51.3.252

46 Canoy D, Wareham N, Luben R, et al. Cigarette smoking and fat distribution in 21,828 British men and women: a population-based study. Obes Res 2005;13:1466-75. doi:10.1038/oby.2005.177

47 Leite ML, Nicolosi A. Lifestyle correlates of anthropometric estimates of body adiposity in an Italian middle-aged and elderly population: a covariance analysis. Int J Obes (Lond) 2006;30:926-34. doi:10.1038/sj.ijo.0803239

\section{Supplementary figures and tables}

